\title{
Inverted spectroscopy and interferometry for quantum-state reconstruction of systems with SU(2) symmetry
}

\author{
C. Briti and A. Mannt \\ Department of Physics, Technion-Israel Institute of Technology, Haifa 32000, Israel
}

\begin{abstract}
We consider how the conventional spectroscopic and interferometric schemes can be rearranged to serve for reconstructing quantum states of physical systems possessing $\mathrm{SU}(2)$ symmetry. The discussed systems include a collection of two-level atoms, a two-mode quantized radiation field with a fixed total number of photons, and a single laser-cooled ion in a two-dimensional harmonic trap with a fixed total number of vibrational quanta. In the proposed rearrangement, the standard spectroscopic and interferometric experiments are inverted. Usually one measures an unknown frequency or phase shift using a system prepared in a known quantum state. Our aim is just the inverse one, i.e., to use a well-calibrated apparatus with known transformation parameters to measure unknown quantum states.

03.65.Bz, 03.65.Fd, 42.50.Dv
\end{abstract}

\section{INTRODUCTION}

The last few years were marked by an outburst of research devoted to the problem of reconstruction of quantum states for various physical systems (see, e.g., Ref. [1] for an extensive list of the literature on the subject). The problem, as stated already in the fifties by Fano [2] and Pauli [3], is to determine the density matrix $\rho$ from information obtained by a set of measurements performed on an ensemble of identically prepared systems. Significant theoretical and experimental progress has been achieved during the last decade in the reconstruction of quantum states of the light field [A. Also, numerous works were devoted to reconstruction methods for other physical systems. Most recently, a general theory of quantum-state reconstruction for physical systems with Lie-group symmetries was developed [1].

In the present work we consider state-reconstruction methods for some quantum systems possessing $\mathrm{SU}(2)$ symmetry. The principal procedure for the reconstruction of spin states was recently presented by Agarwal [5]. A similar approach was also proposed by Dodonov and Man'ko [6], while the basic idea underlying this method goes back to the pioneering work by Royer [7]. In brief, one applies a phase-space displacement [specifically, a rotation in the $\mathrm{SU}(2)$ case] to the initial quantum state and then measures the probability to find the displaced system in a specific state (the so-called "quantum ruler" state). Repeating this procedure with identically prepared systems for many phase-space points [many rotation angles in the $\mathrm{SU}(2)$ case], one determines a function on the phase space (the so-called operational phase-space probability distribution [8 10]). In particular, by measuring the population of the ground state, one obtains the so-called $Q$ function. The information contained in the operational phase-space probability distribution is sufficient to completely reconstruct the unknown density matrix of the initial quantum state. A general grouptheoretical description of this method and some exam- ples, including $\mathrm{SU}(2)$, are presented in Ref. [1].

The aim of the present paper is to study how the general state-reconstruction procedure outlined above can be implemented in practice for a number of specific physical systems with SU(2) symmetry. Three systems are considered: a collection of two-level atoms, a two-mode quantized radiation field with a fixed total number of photons, and a single laser-cooled ion in a two-dimensional harmonic trap with a fixed total number of vibrational quanta. We show that a simple rearrangement of conventional spectroscopic and interferometric schemes enables one to measure unknown quantum states of these systems.

\section{RECONSTRUCTION OF QUANTUM STATES FOR SYSTEMS WITH SU(2) SYMMETRY}

We start with some basic properties of $\mathrm{SU}(2)$ which is the dynamical symmetry group for the angular momentum or spin and for many other systems (e.g., a collection of two-level atoms, the Stokes operators describing the polarization of the quantized light field, two light modes with a fixed total photon number, etc.). The su(2) simple Lie algebra consists of the three operators $\left\{J_{x}, J_{y}, J_{z}\right\}$,

$$
\left[J_{p}, J_{r}\right]=i \epsilon_{p r t} J_{t} .
$$

The Casimir operator is a constant times the unit operator, $\mathbf{J}^{2}=j(j+1) I$, for any unitary irreducible representation of the $\mathrm{SU}(2)$ group; so the representations are labeled by the single index $j$ that takes the values $j=0,1 / 2,1,3 / 2, \ldots$. The representation Hilbert space $\mathcal{H}_{j}$ is spanned by the complete orthonormal basis $\{|j, \mu\rangle\}$ (where $\mu=j, j-1, \ldots,-j$ ):

$$
\mathbf{J}^{2}|j, \mu\rangle=j(j+1)|j, \mu\rangle, \quad J_{z}|j, \mu\rangle=\mu|j, \mu\rangle .
$$

In the following we assume that the state $|\psi\rangle$ of the system belongs to $\mathcal{H}_{j}$ (or, for mixed states, that the density 
matrix $\rho$ is an operator on $\mathcal{H}_{j}$ ). Group elements can be parametrized using the Euler angles $\alpha, \beta, \gamma$ :

$$
g=g(\alpha, \beta, \gamma)=e^{i \alpha J_{z}} e^{i \beta J_{y}} e^{i \gamma J_{z}} .
$$

We will employ two very useful concepts: the phase space (which is the group coset space of maximum symmetry) and the coherent states (each point of the phase space corresponds to a coherent state). For $\mathrm{SU}(2)$, the phase space is the unit sphere $\mathbb{S}^{2}=\mathrm{SU}(2) / \mathrm{U}(1)$, and each coherent state is characterized by a unit vector [11, 12]

$$
\mathbf{n}=(\sin \theta \cos \phi, \sin \theta \sin \phi, \cos \theta) .
$$

Specifically, the coherent states $|j ; \mathbf{n}\rangle$ are given by the action of the group element

$$
g(\mathbf{n})=e^{-i \phi J_{z}} e^{-i \theta J_{y}}
$$

on the highest-weight state $|j, j\rangle$ :

$$
\begin{aligned}
|j ; \mathbf{n}\rangle=g(\mathbf{n})|j, j\rangle= & \sum_{\mu=-j}^{j}\left(\begin{array}{c}
2 j \\
j+\mu
\end{array}\right)^{1 / 2} \cos ^{j+\mu}(\theta / 2) \\
& \times \sin ^{j-\mu}(\theta / 2) e^{-i \mu \phi}|j, \mu\rangle .
\end{aligned}
$$

An important property of the coherent states is the resolution of the identity:

$$
\frac{2 j+1}{4 \pi} \int_{\mathbb{S}^{2}} d \mathbf{n}|j ; \mathbf{n}\rangle\langle j ; \mathbf{n}|=I,
$$

where $d \mathbf{n}=\sin \theta d \theta d \phi$.

A possible procedure for the quantum-state reconstruction is as follows [1, [. [6]. First, the system, whose initial state is described by the density matrix $\rho$, is displaced in the phase space:

$$
\rho \rightarrow \rho(\mathbf{n})=g^{-1}(\mathbf{n}) \rho g(\mathbf{n}), \quad \mathbf{n} \in \mathbb{S}^{2} .
$$

Then one measures the probability to find the displaced system in one of the states $|j, \mu\rangle$ (e.g., in the highest state $|j, j\rangle)$. This probability

$$
p_{\mu}(\mathbf{n})=\langle j, \mu|\rho(\mathbf{n})| j, \mu\rangle
$$

(which is sometimes called the operational phase-space probability distribution) can be formally considered as the expectation value

$$
p_{\mu}(\mathbf{n})=\operatorname{Tr}\left[\rho \Gamma_{\mu}(\mathbf{n})\right]
$$

of the so-called displaced projector

$$
\Gamma_{\mu}(\mathbf{n})=g(\mathbf{n})|j, \mu\rangle\langle j, \mu| g^{-1}(\mathbf{n}) .
$$

Repeating this procedure (with a large number of identically prepared systems) for a large number of phase-space points $\mathbf{n}$, one can determine the function $p_{\mu}(\mathbf{n})$.

Knowledge of the function $p_{\mu}(\mathbf{n})$ is sufficient for the reconstruction of the initial density matrix $\rho$. We can use the following expansion for the density matrix (such an expansion exists for any operator on $\mathcal{H}_{j}$ ):

$$
\rho=\sum_{l=0}^{2 j} \sum_{m=-l}^{l} \mathcal{R}_{l m} D_{l m}, \quad \mathcal{R}_{l m}=\operatorname{Tr}\left(\rho D_{l m}^{\dagger}\right) .
$$

Here, $D_{l m}$ are the so-called tensor operators (also known in the context of angular momentum as the Fano multipole operators [13]),

$$
D_{l m}=\sqrt{\frac{2 l+1}{2 j+1}} \sum_{k, q=-j}^{j}\langle j, k ; l, m \mid j, q\rangle|j, q\rangle\langle j, k|,
$$

where $\left\langle j_{1}, m_{1} ; j_{2}, m_{2} \mid j, m\right\rangle$ are the Clebsch-Gordan coefficients. Now, one can reconstruct the density matrix by using the relation [ㄴ.5]

$$
\mathcal{R}_{l m}=\frac{\sqrt{(2 j+1) / 4 \pi}}{\langle j, \mu ; l, 0 \mid j, \mu\rangle} \int_{\mathbb{S}^{2}} d \mathbf{n} p_{\mu}(\mathbf{n}) Y_{l m}^{*}(\mathbf{n}),
$$

where $Y_{l m}(\mathbf{n})$ are the spherical harmonics. Other ways to deduce the density matrix from the measured probabilities $p_{\mu}(\mathbf{n})$ were also proposed 14].

Let us also consider the useful concept of phase-space quasiprobability distributions (QPDs). In the $\mathrm{SU}(2)$ case, one can introduce an $s$-parametrized family of the QPDs [1, 15, 16]

$$
P(\mathbf{n} ; s)=\sum_{l=0}^{2 j} \sum_{m=-l}^{l} \frac{\sqrt{4 \pi /(2 j+1)}}{\langle j, j ; l, 0 \mid j, j\rangle^{s}} \mathcal{R}_{l m} Y_{l m}(\mathbf{n}) .
$$

For $s=0$, we have the $\mathrm{SU}(2)$ equivalent of the Wigner function,

$$
W(\mathbf{n})=\sqrt{\frac{4 \pi}{2 j+1}} \sum_{l=0}^{2 j} \sum_{m=-l}^{l} \mathcal{R}_{l m} Y_{l m}(\mathbf{n}) .
$$

For $s=1$, we obtain the $\mathrm{SU}(2)$ equivalent of the GlauberSudarshan function (also known as Berezin's contravariant symbol), $P(\mathbf{n})$, whose defining property is

$$
\rho=\frac{2 j+1}{4 \pi} \int_{\mathbb{S}^{2}} d \mathbf{n} P(\mathbf{n})|j ; \mathbf{n}\rangle\langle j ; \mathbf{n}| .
$$

The function which is probably the most important for the reconstruction problem is the $\mathrm{SU}(2)$ equivalent of the Husimi function (also known as Berezin's covariant symbol),

$$
Q(\mathbf{n})=\langle j ; \mathbf{n}|\rho| j ; \mathbf{n}\rangle,
$$

obtained for $s=-1$. As is seen from Eq. (8), the function $Q(\mathbf{n})$ gives the probability to find the displaced system in the highest spin state $|j, j\rangle$,

$$
Q(\mathbf{n})=p_{j}(\mathbf{n}) .
$$


Also, one can see that the probability $p_{-j}(\theta, \phi)$ to find the displaced system in the lowest spin state $|j,-j\rangle$ is equal to $Q(\theta+\pi, \phi)$. More generally, any one of the QPDs can be reconstructed using the relation [1]

$$
\begin{aligned}
& P(\mathbf{n} ; s)=\frac{2 j+1}{4 \pi} \int_{\mathbb{S}^{2}} d \mathbf{n}^{\prime} K_{\mu, s}^{-}\left(\mathbf{n}, \mathbf{n}^{\prime}\right) p_{\mu}\left(\mathbf{n}^{\prime}\right), \\
& K_{\mu, s}^{-}\left(\mathbf{n}, \mathbf{n}^{\prime}\right)=\sum_{l=0}^{2 j} \frac{2 l+1}{2 j+1} \frac{\langle j, j ; l, 0 \mid j, j\rangle^{-s}}{\langle j, \mu ; l, 0 \mid j, \mu\rangle} P_{l}\left(\mathbf{n} \cdot \mathbf{n}^{\prime}\right),
\end{aligned}
$$

where $P_{l}(x)$ are the Legendre polynomials. For $s=-1$ and $\mu=j$ we recover the relation (18).

\section{GENERAL DESCRIPTION OF EXPERIMENTAL SCHEMES}

\section{A. Spectroscopy and interferometry}

Quantum transformations which constitute the basic operations in spectroscopic and interferometric measurements can be conveniently described as rotations in an abstract 3-dimensional space. In this description, the system is characterized by the vector $\mathbf{J}=\left(J_{x}, J_{y}, J_{z}\right)^{T}$, where the three operators $J_{x}, J_{y}$, and $J_{z}$ satisfy the $\operatorname{su}(2)$ algebra (11).

A spectroscopic or interferometric process is usually described in the Heisenberg picture as a unitary transformation

$$
\mathbf{J}_{\text {out }}=U\left(\vartheta_{1}, \vartheta_{2}, \varphi\right) \mathbf{J} U^{\dagger}\left(\vartheta_{1}, \vartheta_{2}, \varphi\right)=\mathrm{U}\left(\vartheta_{1}, \vartheta_{2}, \varphi\right) \mathbf{J}
$$

where $\mathrm{U}\left(\vartheta_{1}, \vartheta_{2}, \varphi\right)$ is a $3 \times 3$ transformation (rotation) matrix, and $\vartheta_{1}, \vartheta_{2}, \varphi$ are transformation parameters (rotation angles). A standard transformation consists of three steps:

(i) rotation around the $\hat{\mathbf{y}}$ axis by $\vartheta_{1}$, with the transformation matrix $\mathrm{R}_{y}\left(\vartheta_{1}\right)$,

(ii) rotation around the $\hat{\mathbf{z}}$ axis by $\varphi$, with the transformation matrix $\mathrm{R}_{z}(\varphi)$,

(iii) rotation around the $\hat{\mathbf{y}}$ axis by $\vartheta_{2}$, with the transformation matrix $\mathrm{R}_{y}\left(\vartheta_{2}\right)$.

The overall transformation performed on $\mathbf{J}$ is

$$
\mathrm{U}(\theta, \phi)=\mathrm{R}_{y}\left(\vartheta_{2}\right) \mathrm{R}_{z}(\varphi) \mathrm{R}_{y}\left(\vartheta_{1}\right)
$$

This transformation is slightly more general than those routinely made in spectroscopy and interferometry. The usual choice is $\vartheta_{2}=-\vartheta_{1}= \pm \pi / 2$, so $\mathrm{U}=\mathrm{R}_{x}( \pm \varphi)$, respectively, while $\varphi$ is the parameter to be estimated in the experiment. In the Schrödinger picture, the density matrix of the system transforms as

$$
\rho_{\text {out }}=U^{\dagger}\left(\vartheta_{1}, \vartheta_{2}, \varphi\right) \rho U\left(\vartheta_{1}, \vartheta_{2}, \varphi\right)
$$

where the transformation operator is

$$
U\left(\vartheta_{1}, \vartheta_{2}, \varphi\right)=e^{i \vartheta_{1} J_{y}} e^{i \varphi J_{z}} e^{i \vartheta_{2} J_{y}}
$$

Now, the aim is to measure the value of $\varphi$ which is proportional to the transition frequency in a spectroscopic experiment or to the optical path difference between the two arms of an interferometer. The information on $\varphi$ is inferred from the measurement of the observable $J_{z}$ at the output. The quantum uncertainty in the estimation of $\varphi$ is

$$
\Delta \varphi=\frac{\Delta J_{z \text { out }}}{\left|\partial\left\langle J_{z \text { out }}\right\rangle / \partial \varphi\right|},
$$

where the expectation values are taken over the initial quantum state of the system. This state is assumed to be known, so one can estimate the value of $\varphi$ and the corresponding uncertainty.

\section{B. Reconstruction of the initial state}

In this paper we consider how to use the spectroscopic or interferometric arrangement for the inverse purpose, i.e., for the measurement of an unknown initial quantum state by means of a large number of transformations with known parameters.

As discussed in Sec. II, the first part of the reconstruction procedure is the phase-space displacement of Eq. (7). With the phase space being the sphere, this displacement is just a rotation produced by the operator $g(\mathbf{n})$ of Eq. (4). Now, compare this rotation with the one made during a spectroscopic or interferometric experiment, as given by Eqs. (23) and (24). One can immediately conclude that the phase-space displacement needed for the $\mathrm{SU}(2)$ state-reconstruction procedure can be neatly implemented by means of the spectroscopic and interferometric techniques. One only needs to omit the first rotation (i.e., take $\vartheta_{1}=0$ ), and recognize the two spherical angles as:

$$
\theta=-\vartheta_{2}, \quad \phi=-\varphi
$$

After the rotation $g(\mathbf{n})$ is made, one should measure the probability $p_{\mu}(\mathbf{n})$ to find the displaced system in the state $|j, \mu\rangle$. Perhaps the most convenient choice is to measure the population of the lowest state $|j,-j\rangle$, which is usually the ground state of the system (e.g., this state corresponds to the case where all the atoms are unexcited; in the atomic case such a measurement can be made by monitoring the resonant fluorescence for an auxiliary dipole transition). This procedure should be repeated for many phase-space points $\mathbf{n}$ with a large number of identically prepared systems, thereby determining the function $p_{\mu}(\mathbf{n})$ (e.g., for $\mu=j$ or $\mu=-j$ ). According to the formalism presented in Sec. II, this information is sufficient to reconstruct the initial quantum state. 


\section{COLLECTIONS OF TWO-LEVEL ATOMS}

In Ramsey spectroscopy [17] one deals with a collection of $N$ two-level systems (usually atoms or ions) interacting with classical light fields. One can equivalently describe this physical situation as the interaction of $N$ spin- $\frac{1}{2}$ particles with classical magnetic fields. Denoting by $\mathbf{S}_{i}$ the spin of $i$ th particle, one can use the collective spin operators:

$$
\mathbf{J}=\sum_{i=1}^{N} \mathbf{S}_{i}
$$

The orthonormal basis $\{|j, \mu\rangle\}$ consists of the symmetric Dicke states [18]:

$$
|j, \mu\rangle=\left(\begin{array}{c}
N \\
p
\end{array}\right)^{-1 / 2} \sum \prod_{k=1}^{p}|+\rangle_{l_{k}} \prod_{l \neq l_{k}}|-\rangle_{l},
$$

where $|+\rangle_{l}$ and $|-\rangle_{l}$ are the upper and lower states, respectively, of the $l$ th atom, and the summation is over all possible permutations of $N$ atoms. If only symmetric states are considered, then the "cooperative number" $j$ is equal to $N / 2$ and $p=\mu+j$ is just the number of excited atoms. Usually the atoms (ions) are far enough apart so their wave functions do not overlap and the direct dipoledipole coupling or other direct interactions between the atoms may be neglected.

In the spin formulation (see, e.g., Ref. [19] for a very good description), the magnetic moment $\boldsymbol{\mu}=\mu_{0} \mathbf{S}$ is associated with each particle. If a uniform external magnetic field $\mathbf{B}_{0}=B_{0} \hat{\mathbf{z}}$ is applied, the Hamiltonian for each particle is given by

$$
H_{0}=-\boldsymbol{\mu} \cdot \mathbf{B}_{0}=\hbar \omega_{0} S_{z}
$$

where $\hbar \omega_{0}=-\mu_{0} B_{0}$ is the separation in energy between the two levels. The corresponding Heisenberg equation for the collective spin operator is

$$
\partial \mathbf{J} / \partial t=\boldsymbol{\omega}_{0} \times \mathbf{J}
$$

where $\boldsymbol{\omega}_{0}=\omega_{0} \hat{\mathbf{z}}$. Then one applies the so-called clock radiation which is a classical field of the form

$$
\mathbf{B}_{\perp}=B_{\perp}(\hat{\mathbf{y}} \cos \omega t-\hat{\mathbf{x}} \sin \omega t)
$$

where $\omega \simeq \omega_{0}$ and we assume $\omega_{0}>0$. In the reference frame that rotates at frequency $\omega$, the collective spin $\mathbf{J}$ interacts with the effective field

$$
\mathbf{B}=B_{r} \hat{\mathbf{z}}+B_{\perp} \hat{\mathbf{y}}
$$

where $B_{r}=B_{0}\left(\omega_{0}-\omega\right) / \omega_{0}$. In the rotating frame, the Hamiltonian is $H=-\mu_{0} \mathbf{J} \cdot \mathbf{B}$, and the Heisenberg equation for $\mathbf{J}$ is

$$
\partial \mathbf{J} / \partial t=\boldsymbol{\omega}^{\prime} \times \mathbf{J}
$$

where $\boldsymbol{\omega}^{\prime}=\left(\omega_{0}-\omega\right) \hat{\mathbf{z}}+\omega_{\perp} \hat{\mathbf{y}}$ and $\omega_{\perp}=-\mu_{0} B_{\perp} / \hbar$.

The Ramsey method breaks the evolution time of the system into three parts. In the first part $B_{\perp}$ is nonzero and constant with value $B_{1}$ during the time interval $0 \leq t \leq t_{\vartheta}$. During this period (the first Ramsey pulse), $\mathbf{B}=B_{r} \hat{\mathbf{z}}+B_{1} \hat{\mathbf{y}} \simeq B_{1} \hat{\mathbf{y}}$, where we made the assumption $\left|B_{1}\right| \gg\left|B_{r}\right|$, i.e., $\left|\omega_{1}\right| \gg\left|\omega_{0}-\omega\right|$, with $\omega_{1}=-\mu_{0} B_{1} / \hbar$. Therefore, in the rotating frame of Eq. (33), J rotates around the $\hat{\mathbf{y}}$ axis by the angle $\vartheta_{1}=\omega_{1} t_{\vartheta}$. During the second period, of duration $T$, (usually $T \gg t_{\vartheta}$ ), $B_{\perp}$ is zero, so $\mathbf{B}=B_{r} \hat{\mathbf{z}}$, and $\mathbf{J}$ rotates around the $\hat{\mathbf{z}}$ axis by the angle $\varphi=\left(\omega_{0}-\omega\right) T$. The third period is exactly as the first one but with the field $B_{\perp}=B_{2}$ and the corresponding angular frequency $\omega_{2}=-\mu_{0} B_{2} / \hbar$. This gives a rotation around the $\hat{\mathbf{y}}$ axis by the angle $\vartheta_{2}=\omega_{2} t_{\vartheta}$. These three Ramsey pulses provide the rotations we described in Sec. III A (usually, $\vartheta_{1}=\vartheta_{2}=\pi / 2$ ).

The aim of spectroscopic experiments is to measure the transition frequency $\omega_{0}$ (which is equivalent to the measurement of $\varphi$, as $\omega$ and $T$ are determined by the experimenter). Usually, one measures the number of atoms in the upper state $|+\rangle$,

$$
N_{\text {out }}=J_{z \text { out }}+N / 2
$$

and thus obtains the information about the angle $\varphi$ or, equivalently, about the frequency $\omega_{0}$. Of course, in order to infer this information one should know the initial quantum state of the system. The measurement sensitivity, as seen from Eq. (25), also depends on the initial quantum state.

In the state-reconstruction procedure, the first Ramsey pulse should be omitted, while the second and third pulses produce the desired phase-space displacement $g^{\dagger}(\mathbf{n})$. After the displacement is completed, one should measure the probability to find the system in one of the states $|j, \mu\rangle$, for example, measure the population of the ground state $|j,-j\rangle$ or of the most excited state $|j, j\rangle$. This measurement can be made by driving a dipole transition to an auxiliary atomic level and then observing the resonance fluorescence. The phase space is scanned by repeating the measurement with many identically prepared systems for various durations of the Ramsey pulses, $T$ and $t_{\vartheta}$. Of course, the apparatus should be first calibrated by measuring the transition frequency $\omega_{0}$.

\section{TWO-MODE LIGHT FIELDS}

The basic device employed in a passive optical interferometer is a beam splitter (a partially transparent mirror). A Mach-Zehnder interferometer consists of two beam splitters and its operation is as follows. Two light modes (with boson annihilation operators $a_{1}$ and $a_{2}$ ) are mixed by the first beam splitter, accumulate phase shifts $\varphi_{1}$ and $\varphi_{2}$, respectively, and then they are once again mixed by the second beam splitter. Photons in the output modes are counted by two photodetectors. In fact, 
a Michelson interferometer works in the same way, but due to its geometric layout the two beam splitters may coincide.

Each beam splitter has two input and two output ports. Let $\mathbf{a}=\left(a_{1}, a_{2}\right)^{T}$ and $\mathbf{b}=\left(b_{1}, b_{2}\right)^{T}$ be the columnvectors of the boson operators of the input and output modes, respectively. Then, in the Heisenberg picture, the action of the beam splitter is described by the transformation

$$
\mathbf{b}=\mathrm{B} \mathbf{a}
$$

where $B$ is a $2 \times 2$ matrix. For a lossless beam splitter $B$ must be unitary, thereby assuring the energy (photon number) conservation. A possible form of $B$ is

$$
\mathrm{B}(\vartheta)=\left(\begin{array}{cc}
\cos (\vartheta / 2) & -\sin (\vartheta / 2) \\
\sin (\vartheta / 2) & \cos (\vartheta / 2)
\end{array}\right),
$$

with $T=\cos ^{2}(\vartheta / 2)$ and $R=\sin ^{2}(\vartheta / 2)$ being the transmittance and reflectivity, respectively. When the two light modes accumulate phase shifts $\varphi_{1}$ and $\varphi_{2}$, respectively, the corresponding transformation is

$$
\mathbf{b}=\mathrm{P} \mathbf{a}, \quad \mathrm{P}=\left(\begin{array}{cc}
e^{i \varphi_{1}} & 0 \\
0 & e^{i \varphi_{2}}
\end{array}\right) .
$$

The group-theoretic description of the interferometric process [20] is based on the Schwinger realization of the $\mathrm{su}(2)$ algebra:

$$
\begin{aligned}
& J_{x}=\left(a_{1}^{\dagger} a_{2}+a_{2}^{\dagger} a_{1}\right) / 2, \\
& J_{y}=-i\left(a_{1}^{\dagger} a_{2}-a_{2}^{\dagger} a_{1}\right) / 2, \\
& J_{z}=\left(a_{1}^{\dagger} a_{1}-a_{2}^{\dagger} a_{2}\right) / 2 .
\end{aligned}
$$

Actions of the interferometer elements (mixing by the beam splitters and phase shifts) can be represented as rotations of the column-vector $\mathbf{J}=\left(J_{x}, J_{y}, J_{z}\right)^{T}$. The beam-splitter transformation of Eq. (36) is represented by rotation $\mathrm{R}_{y}(\vartheta)$ around the $\hat{\mathbf{y}}$ axis by the angle $\vartheta$, and the phase shift of Eq. (37) is represented by rotation $\mathrm{R}_{z}(\varphi)$ around the $\hat{\mathbf{z}}$ axis by the angle $\varphi=\varphi_{2}-\varphi_{1}$. Now, if the transmittances of the two beam splitters are $T_{1}=\cos ^{2}\left(\vartheta_{1} / 2\right)$ and $T_{2}=\cos ^{2}\left(\vartheta_{2} / 2\right)$, respectively, then the interferometer action is given by the three rotations described in Sec. III A. (Usually, one uses 50-50 beam splitters, so $\vartheta_{1}=-\vartheta_{2}=\pi / 2$.)

Interferometers are constructed to measure the relative phase shift $\varphi$, which is proportional to the optical path difference between the two arms. Usually, one measures the difference between the photocurrents due to the two output light beams. This quantity is proportional to the photon-number difference at the output, $q_{\text {out }}=2 J_{z \text { out }}$. If the input state of light is known, then the measurement of $q_{\text {out }}$ can be used to infer the phase shift $\varphi$ and estimate the measurement error due to the quantum fluctuations of the light field.

A simple calculation gives $\mathbf{J}^{2}=(N / 2)(1+N / 2)$, where $N=a_{1}^{\dagger} a_{1}+a_{2}^{\dagger} a_{2}$ is the total number of photons in the two modes. If $N$ has a fixed value for the input state of the two-mode light field, then this state belongs to the Hilbert space $\mathcal{H}_{j}$ of a specific $\mathrm{SU}(2)$ representation with $j=N / 2$. Because $N$ is the $\mathrm{SU}(2)$ invariant, this state will remain in $\mathcal{H}_{j}$ during the interferometric process. Such input states of the two-mode light field can be reconstructed using a rearrangement of the interferometric scheme, according to the general procedure described in Secs. II and III B.

The phase-space displacement $g^{\dagger}(\mathbf{n})$ needed for the state-reconstruction procedure can be implemented by using an interferometer without the first beam splitter. Then one should measure the probability $p_{\mu}(\mathbf{n})$ to find the output light in one of the states $|j, \mu\rangle$. Note that these states are given by

$$
|j, \mu\rangle=|j+\mu\rangle_{1} \otimes|j-\mu\rangle_{2}
$$

in the terms of the Fock states of the two light modes. So, $\mu$ is just one half of the photon-number difference measured at the output. Averaging over many measurements, one obtains the probabilities $p_{\mu}(\mathbf{n})$. For example, $p_{j}(\mathbf{n})$ is the probability that all photons exit in the first output beam while the number of photons in the second output beam is zero. The measurement should be repeated with identically prepared input light beams for many phase-space displacements. This means that one needs a well-calibrated apparatus which can be tuned for various values of the relative phase shift $\varphi$. These phase shifts can be conveniently produced by moving a mirror with a precise electro-mechanical system. Various values of the angle $\vartheta_{2}$ can be realized using a collection of partially transparent mirrors with different reflectivities for the second beam splitter. An alternative possibility is to use the dependence of the reflectivity on the angle of incidence for light polarized in the plane of incidence.

In general, the state reconstruction for two-mode light fields is a tedious task, because the corresponding Hilbert space is very large [21 25]. Obviously, this task can be greatly simplified for the subclass of two-mode states with a fixed total number of photons, by means of the reconstruction method presented here. However, this method is in principle suitable also for other two-mode states as well. In general, the whole Hilbert space of the two-mode system can be decomposed as

$$
\mathcal{H}=\bigoplus_{j} \mathcal{H}_{j}
$$

The method of inverted interferometry enables one to reconstruct the part of the density matrix corresponding to each irreducible subspace $\mathcal{H}_{j}$. One case for which our method is applicable is the subclass of states, whose density matrices are block-diagonal in terms of the decomposition (40). This means that the corresponding operator can be written as

$$
\rho=\sum_{j} \rho_{j}
$$


where $\rho_{j}$ is an operator on $\mathcal{H}_{j}$. Each component $\rho_{j}$ evolves independently during the phase-space displacement; hence the state of the whole system can be measured by reconstructing all invariant components $\rho_{j}$. The other case for which our method works is the subclass of pure states,

$$
|\psi\rangle=\sum_{j}\left|\psi_{j}\right\rangle, \quad\left|\psi_{j}\right\rangle=\sum_{\mu=-j}^{j} c_{j \mu}|j, \mu\rangle .
$$

Then the density matrix can be written as

$$
\rho=\sum_{j}\left|\psi_{j}\right\rangle\left\langle\psi_{j}\left|+\sum_{j \neq j^{\prime}}\right| \psi_{j}\right\rangle\left\langle\psi_{j^{\prime}}\right| .
$$

The populations of the states $|j, \mu\rangle$ are unaffected by the second term in (43), and one can reconstruct all invariant components $\rho_{j}=\left|\psi_{j}\right\rangle\left\langle\psi_{j}\right|$. This gives information about the state $|\psi\rangle$ of the whole system, except for relative phases between different $\left|\psi_{j}\right\rangle$. From the technical point of view, each measurement of the photon-number difference $2 \mu$, needed to determine the probabilities $p_{\mu}(\mathbf{n})$, should be accompanied by a measurement of the photon-number sum $N=2 j$, in order to determine to which invariant subspace $\mathcal{H}_{j}$ does the detected value of $\mu$ correspond. Consequently, one needs to make many more measurements, in order to accumulate enough data for each value of $j$. A technical problem is that quantum efficiencies of realistic photodetectors are always less then unity. While this problem is not too serious for the measurement of the photon-number difference (as long as both detectors have the same efficiency), it puts a serious limitation on the accuracy of the measurement of the total number of photons.

\section{TWO-DIMENSIONAL VIBRATIONS OF A TRAPPED ION}

As was recently demonstrated by Wineland et al. [26, a single laser-cooled ion in a harmonic trap can be used to simulate various interactions governing many well-known optical processes. In particular, one can simulate transformations produced by elements of a Mach-Zehnder optical interferometer.

Consider a single ion confined in a two-dimensional harmonic trap, with angular frequencies of oscillations in two orthogonal directions $\Omega_{1}$ and $\Omega_{2}$. Two internal states of the ion, $|+\rangle$ and $|-\rangle$, are separated in energy by $\hbar \omega_{0}$. The internal and motional degrees of freedom can be coupled by applying classical laser beams, with electric fields of the form

$$
\mathbf{E}(\mathbf{x}, t)=\mathbf{E}_{0} \cos (\mathbf{k} \cdot \mathbf{x}-\omega t+\Phi) .
$$

For example, one can apply two laser beams to produce stimulated Raman transitions. We denote by $\omega=$ $\omega_{1}-\omega_{2}, \mathbf{k}=\mathbf{k}_{1}-\mathbf{k}_{2}$, and $\Phi=\Phi_{1}-\Phi_{2}$ the differences between the angular frequencies, the wave vectors, and the phases, respectively, of the two applied fields. Then, in the rotating-wave approximation, the interaction Hamiltonian reads

$$
H_{I}=\hbar \kappa \exp [i(\mathbf{k} \cdot \mathbf{x}-\delta t+\Phi)]+\text { H.c., }
$$

where $\delta=\omega-\omega_{0}$ is the frequency detuning, $\mathbf{x}$ is the ion's position relative to its equilibrium, and $\kappa$ is the coupling constant (the Rabi frequency). Each of the two modes of the ion's motion can be modelled by a quantum harmonic oscillator:

$$
x_{r}=x_{0 r}\left(a_{r}+a_{r}^{\dagger}\right), \quad x_{0 r}=\sqrt{\hbar /\left(2 M \Omega_{r}\right)},
$$

where $r=1,2$ and $M$ is the ion's mass. Also, let $\eta_{r}=k_{r} x_{0 r}(r=1,2)$ be the Lamb-Dicke parameters for the two oscillatory modes. It is convenient to use the interaction picture for the ion's motion:

$$
\begin{aligned}
\tilde{H}_{I} & =\exp \left(i H_{0} t / \hbar\right) H_{I} \exp \left(-i H_{0} t / \hbar\right) \\
& =\hbar \kappa e^{i(\Phi-\delta t)} \prod_{r=1,2} \exp \left[i \eta_{r}\left(\tilde{a}_{r}+\tilde{a}_{r}^{\dagger}\right)\right]+\text { H.c. }
\end{aligned}
$$

where $H_{0}$ is the free Hamiltonian for the ion's motion,

$$
H_{0}=\hbar \Omega_{1}\left(a_{1}^{\dagger} a_{1}+\frac{1}{2}\right)+\hbar \Omega_{2}\left(a_{2}^{\dagger} a_{2}+\frac{1}{2}\right),
$$

and $\tilde{a}_{r}=a_{r} \exp \left(-i \Omega_{r} t\right), r=1,2$.

If the coupling constant $\kappa$ is small enough and $\Omega_{1}$ and $\Omega_{2}$ are incommensurate, one can resonantly excite only one spectral component of the possible transitions. For a particular resonance condition $\delta=\Omega_{2}-\Omega_{1}$ (and in the Lamb-Dicke limit of small $\eta_{1}$ and $\eta_{2}$ ), the product in Eq. (46) will be dominated by the single term $\left(i \eta_{1} a_{1}\right)\left(i \eta_{2} a_{2}^{\dagger}\right)$. Therefore, one obtains

$$
\tilde{H}_{I} \approx-\hbar \kappa \eta_{1} \eta_{2}\left(e^{i \Phi} a_{1} a_{2}^{\dagger}+e^{-i \Phi} a_{1}^{\dagger} a_{2}\right) .
$$

Returning to the Schrödinger picture, the total evolution operator reads:

$$
\begin{aligned}
U(t)= & \exp \left(-i H_{0} t / \hbar\right) \exp \left(-i \tilde{H}_{I} t / \hbar\right) \\
= & \exp \left[-i\left(\Omega_{1}+\Omega_{2}\right)(N+1) t / 2\right] \exp \left[i\left(\Omega_{2}-\Omega_{1}\right) J_{z} t\right] \\
& \times \exp \left(2 i \kappa \eta_{1} \eta_{2} J_{\Phi} t\right) .
\end{aligned}
$$

Here, $N=a_{1}^{\dagger} a_{1}+a_{2}^{\dagger} a_{2}$ is the total number of vibrational quanta in the two modes, $J_{\Phi}=J_{x} \cos \Phi+J_{y} \sin \Phi$, and we used the Schwinger realization (38) for the SU(2) generators.

Now, let us consider only such motional states of the ion for which $N$ has a fixed value, i.e., which belong to the irreducible Hilbert space $\mathcal{H}_{j}$ (with $j=N / 2$ ). For these states, the first exponent in (49) will just produce an unimportant phase factor and can be omitted. Clearly, the evolution operator (49) can be used to simulate the action of an optical interferometer, with two vibrational modes of a trapped ion employed instead of two light 
beams. In order to simulate the action of a beam splitter, one should apply the interaction (48) during time $t_{\theta}$ and ensure that $\left|2 \kappa \eta_{1} \eta_{2}\right| \gg\left|\Omega_{2}-\Omega_{1}\right|$, so the effect of the free evolution can be neglected. Then, for $\Phi=\pi / 2$, the evolution operator reads

$$
U_{y}(\theta)=\exp \left(i \theta J_{y}\right), \quad \theta=2 \kappa \eta_{1} \eta_{2} t_{\theta} .
$$

A relative phase shift between the two modes can be produced just by using the free evolution, i.e., with no external laser fields applied. Letting the system evolve freely during time $T$, one obtains

$$
U_{z}(\phi)=\exp \left(i \phi J_{z}\right), \quad \phi=\left(\Omega_{2}-\Omega_{1}\right) T .
$$

It is obvious that applying consequently the transformations (51) and (50) one will produce the phase-space displacement $g^{\dagger}(\mathbf{n})$, employed in the state-reconstruction procedure. The whole phase space can be scanned by repeating the procedure with identically prepared systems for various durations $T$ and $t_{\theta}$. Each phase-space displacement should be followed by the measurement of the probability $p_{\mu}(\mathbf{n})$ to find the system in one of the states $|j, \mu\rangle$. For example, $p_{j}(\mathbf{n})$ is the probability that the first oscillatory mode is excited to the $N$ th level $(N=2 j)$ while the second mode is in the ground state. Such a measurement can be made with the method used recently by the NIST group [27] to reconstruct the one-dimensional motional state of a trapped ion. The principle of this method is as follows. One of the oscillatory modes is coupled to the internal transition $|+\rangle \leftrightarrow|-\rangle$. This is done by applying one classical laser field, so single-photon transitions are excited. This results in an interaction of the Jaynes-Cummings type 28 between the oscillatory mode and the internal transition. Then the population $P_{-}(t)$ of the lower internal state $|-\rangle$ is measured for various values of the interaction time $t$ (as we already mentioned, this measurement can be made by monitoring the resonant fluorescence produced in an auxiliary dipole transition). If $|-\rangle$ is the internal state at $t=0$, then the signal averaged over many measurements is

$$
P_{-}(t)=\frac{1}{2}\left[1+\sum_{n=0}^{\infty} P_{n} \cos \left(2 \Omega_{n, n+1} t\right) e^{-\gamma_{n} t}\right],
$$

where $\Omega_{n, n+1}$ are the Rabi frequencies and $\gamma_{n}$ are the experimentally determined decay constants. This relation allows one to determine the populations $P_{n}$ of the motional eigenstates $|n\rangle$. By virtue of Eq. (39), this gives the populations $p_{\mu}$ of the $\mathrm{SU}(2)$ states $|j, \mu\rangle$ (with $\mu=n-j$ for the first mode and $\mu=j-n$ for the second mode). For example, $p_{-j}$ and $p_{j}$ are given by $P_{0}$ for the first and second modes, respectively.

\section{CONCLUSIONS}

In this paper we presented practical methods for the reconstruction of quantum states for a number of physical systems with $\mathrm{SU}(2)$ symmetry. All these methods employ the same basic idea - the measurement of displaced projectors - which in principle is applicable to any system possessing a Lie-group symmetry. Practical realizations, of course, vary for different physical systems. In our approach, we exploited the fact that transformations applied in conventional spectroscopic and interferometric schemes are, from the mathematical point of view, just rotations. In the context of the $\mathrm{SU}(2)$ group, these rotations constitute phase-space displacements needed to implement a part of the reconstruction procedure. Therefore, the spectroscopic and interferometric measurements can be easily rearranged in order to enable one to determine unknown quantum states for an ensemble of identically prepared systems. As the spectroscopic and interferometric measurements are known for their high accuracy, we hope that the corresponding rearrangements will allow accurate reconstructions of unknown quantum states.

\section{ACKNOWLEDGMENTS}

This work was supported by the Fund for Promotion of Research at the Technion and by the Technion VPR Fund.

* Present address: LIGO Project, California Institute of Technology, Pasadena, CA 91125. Electronic address: cbrif@ligo.caltech.edu

$\dagger \quad$ Electronic address: ady@physics.technion.ac.il

[1] C. Brif and A. Mann, Phys. Rev. A 59, 971 (1999).

[2] U. Fano, Rev. Mod. Phys. 29, 74 (1957).

[3] W. Pauli, in Encyclopedia of Physics, Vol. 5, edited by S. Flügge (Springer, Berlin, 1958), p. 17.

[4] U. Leonhardt, Measuring the Quantum State of Light (Cambridge University Press, Cambridge, England, 1997).

[5] G. S. Agarwal, Phys. Rev. A 57, 671 (1998).

[6] V. V. Dodonov and V. I. Man'ko, Phys. Lett. A 229, 335 (1997).

[7] A. Royer, Phys. Rev. Lett. 55, 2745 (1985); Found. Phys. 19, 3 (1989).

[8] K. Wódkiewicz, Phys. Rev. Lett. 52, 1064 (1984); Phys. Lett. A 115, 304 (1986).

[9] V. Bužek, C. H. Keitel, and P. L. Knight, Phys. Rev. A 51, 2575 (1995); 51, 2594 (1995).

[10] M. Ban, Int. J. Theor. Phys. 38, 2583 (1998); J. Math. Phys. 39, 1744 (1998).

[11] F. T. Arecchi, E. Courtens, R. Gilmore, and H. Thomas, Phys. Rev. A 6, 2211 (1972).

[12] A. M. Perelomov, Commun. Math. Phys. 26, 222 (1972); Generalized Coherent States and Their Applications (Springer, Berlin, 1986). 
[13] U. Fano, Phys. Rev. 90, 577 (1953).

[14] J.-P. Amiet and S. Weigert, preprint quant-ph/9903067 (1999), and references therein. The idea is that the Hilbert space $\mathcal{H}_{j}$ is finite-dimensional, and therefore one can reconstruct the density matrix by measuring the probabilities $p_{\mu}(\mathbf{n})$ only at a finite number of phase-space points.

[15] G. S. Agarwal, Phys. Rev. A 24, 2889 (1981).

[16] J. C. Várilly and J. M. Gracia-Bondía, Ann. Phys. (N.Y.) 190, 107 (1989).

[17] N. F. Ramsey, Molecular Beams (Oxford University Press, London, 1963).

[18] R. Dicke, Phys. Rev. 93, 99 (1954).

[19] D. J. Wineland, J. J. Bollinger, W. M. Itano, and D. J. Heinzen, Phys. Rev. A 50, 67 (1994).

[20] B. Yurke, S. L. McCall, and J. R. Klauder, Phys. Rev. A 33, 4033 (1986).

[21] H. Kühn, D.-G. Welsch, and W. Vogel, Phys. Rev. A 51,
4240 (1995).

[22] M. G. Raymer, D. F. McAlister, and U. Leonhardt, Phys. Rev. A 54, 2397 (1996).

[23] T. Opatrný, D.-G. Welsch, and W. Vogel, Opt. Commun. 134, 112 (1997).

[24] Th. Richter, J. Mod. Opt. 44, 2385 (1997); Phys. Rev. A 55, 4629 (1997).

[25] H. Paul, P. Torma, T. Kiss, and I. Jex, J. Mod. Opt. 44, 2395 (1997).

[26] D. J. Wineland, C. Monroe, W. M. Itano, B. E. King, D. Leibfried, C. Myatt, and C. Wood, Phys. Scr. T76, 147 (1998).

[27] D. Leibfried, D. M. Meekhof, B. E. King, C. Monroe, W. M. Itano, and D. J. Wineland, Phys. Rev. Lett. 77, 4281 (1996).

[28] E. T. Jaynes and F. W. Cummings, Proc. IEEE 51, 89 (1963). 